\section{Biallelic IARS2 mutations presenting as sideroblastic anemia}

Aminoacyl-tRNA synthetases (ARS) are evolutionarily conserved enzymes that catalyze amino acid attachment to their cognate transfer RNA (tRNA), ensuring accuracy of the translation process. Two separate sets of cellular ARS are required, as translation takes place in two distinct compartments, namely cytosol and mitochondria. Eighteen ARS act exclusively in the cytosol (ARS1), 17 act exclusively in the mitochondria (ARS2) and two ARS are bifunctional, as they act in both compartments. Mutations in the nuclear genes encoding ARS2 have emerged as a new group of mitochondrial diseases, inconsistently impairing oxidative phosphorylation. ${ }^{2}$ Among them, pathogenic variants in the IARS2 gene (Online Mendelian Inheritance in Man [OMIM] 612801) have been reported to cause overlapping clinical phenotypes ranging from isolated cataract to a syndromic condition with cataract (CA), growth hormone deficiency $(\mathrm{G})$, sensory neuropathy (S), sensorineural hearing loss (S), and skeletal dysplasia syndrome (CAGSSS) OMIM 616007) and Leigh syndrome (Table).

Here, we report on biallelic pathogenic IARS2 variants in three unrelated siblings presenting with neonatal sideroblastic anemia mimicking Pearson syndrome.

Patient 1, the third child of non-consanguineous healthy parents of French origin, was born after a normal full-term pregnancy with normal birth parameters. His older sister and brother are healthy (Figure 2). At birth, he presented with severe sideroblastic anemia (hemoglobin $5.1 \mathrm{~g} / \mathrm{dL}$, normal $>14.5 \mathrm{~g} / \mathrm{dL}$ ) with normal mean corpuscular volume (MCV). Myelogram showed $2 \%$ of ring sider- oblasts (Figure 1D). Search for B19 parvovirus, Coombs test and Kleinhauer test were negative. Plasma B12 and folate were normal. He required two red blood cell transfusions during the first month and thrombopenia reached a nadir of 80.000 platelets $/ \mathrm{mm}^{3}$ normal $>150.000$ platelets $\left./ \mathrm{mm}^{3}\right)$. Plasma lactate $(3.8-5.3 \mathrm{mmol} / \mathrm{L}$, normal $<2 \mathrm{mmol} / \mathrm{L})$, lactate/pyruvate ratios $(33$, normal $<20)$ and cerebrospinal fluid (CSF) lactate were elevated (3.9 $\mathrm{mmol} / \mathrm{L}$, normal $<2 \mathrm{mmol} / \mathrm{L}$ ). The child had exocrine pancreatic dysfunction with decreased fecal elastase (75 $\mu \mathrm{g} / \mathrm{g}$, normal 200-500 $\mu \mathrm{g} / \mathrm{g}$ ) and hypoparathyroidism (plasma calcium $1.24 \mathrm{mmol} / \mathrm{L}$, normal 2.2-2.7 $\mathrm{mmol} / \mathrm{L}$; plasma phosphate $2.8 \mathrm{mmol} / \mathrm{L}$, normal 1.12-1.45 $\mathrm{mmol} / \mathrm{L}$; parathormone $3 \mathrm{pg} / \mathrm{mL}$, normal: $10-55 \mathrm{pg} / \mathrm{mL}$ ). $\mathrm{He}$ presented with congenital bilateral cataract, axial hypotonia, peripheral dystonia and motor delay. At 6 months, he developed pharmaco-resistant infantile spasms with hypsarrhythmia on electroencephalogram (EEG) and vigabatrin and topiramate were started. Metabolic work-up, including plasma amino acids and liver enzymes (aspartate amino transferase/alanine amino transferase [ASAT/ALAT]), urinary organic acids and skeletal X-ray were normal. Brain magnetic resonance imaging (MRI) (3 months) was normal except for a lactate peak on spectroscopy (Figure 1A). The child died at 16 months due to respiratory distress in a context of inhalation pneumonia.

A next-generation sequencing panel targeting genes involved in mitochondrial disorders showed two compound heterozygous IARS2 variants: a novel nonsense variant inherited from his father (c.891G>A; p. Trp297*), predicted to result in either nonsense-mediated (NMD) or loss of the terminal two thirds of the protein including
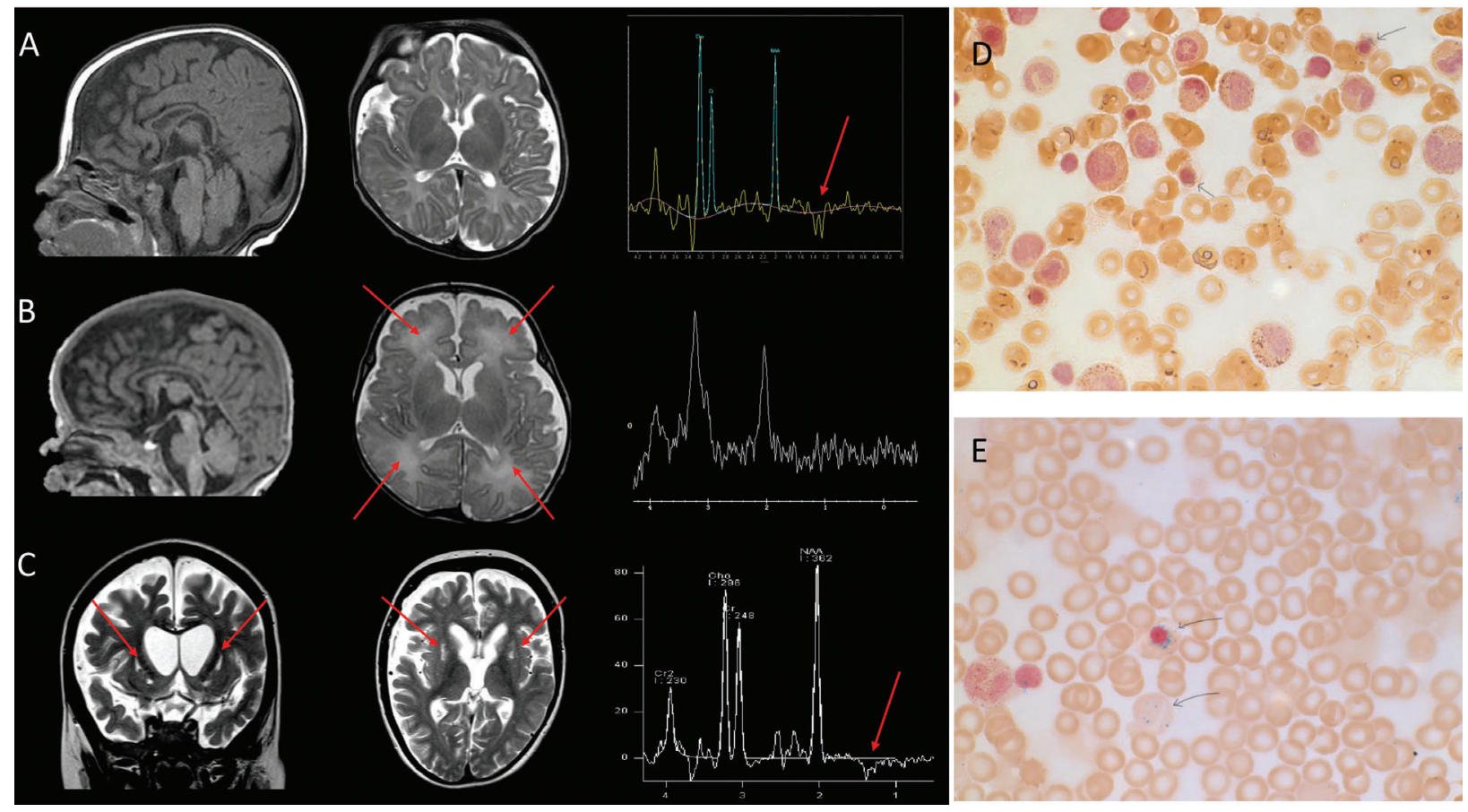

Figure 1. Brain magnetic resonance imaging anomalies in three patients carrying biallelic pathogenic IARS2 variants and bone marrow aspiration from patients 1 and 2. (A) Patient 1 ( 3 months): sagittal T1, axial T2 weighted images and magnetic resonance spectroscopy (MRS) showing no anomalies other than a lactate peak (arrow). (B) Patient 2 (2 months): sagittal T1, axial T2 weighted images and MRS showing no lactate peak but mild and diffuse white matter hyperintensities (arrows). (C) Patient 3 (7 years): coronal T2, axial T2 weighted images and MRS showing bilateral cavitations in putamen characteristic of Leigh disease (arrows), frank and diffuse cerebral atrophy, white matter loss and a lactate peak. (D, E) Bone marrow aspiration from patient 1 (D) and 2 (E) showing ring sideroblasts on iron stain. 
Table 1. Clinical, neuroimaging and molecular findings in patients carrying pathogenic IARS2 variants.

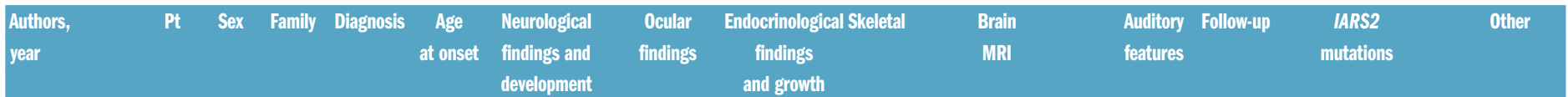

\begin{tabular}{|c|c|c|c|c|c|c|c|c|c|c|c|c|c|c|}
\hline \multirow[t]{4}{*}{$\begin{array}{l}\text { Schwartzentruber } \\
\text { et al., } 2014\end{array}$} & 1 & $\mathrm{~F}$ & 1 & CAGSSS & Birth & $\begin{array}{c}\text { DD, distal } \\
\text { sensory } \\
\text { neuropathy } \\
(9,5 \mathrm{y})\end{array}$ & $\begin{array}{c}\text { Cataract } \\
(17 \mathrm{~m}), \\
\text { corneal } \\
\text { opacification } \\
\text { (5y), orbital } \\
\text { myopathy (32 y) }\end{array}$ & $\begin{array}{c}\text { GH } \\
\text { deficiency } \\
(15 \mathrm{y}) \quad \mathrm{d} \\
\text { Short } \mathrm{S} \\
\text { stature }\end{array}$ & $\begin{array}{l}\text { Bilateral } \\
\text { hip } \\
\text { dislocation, } \\
\text { SEMD (2 y) }\end{array}$ & $\begin{array}{c}\text { Atrophied } \\
\text { pituitary } \\
\text {, adenohypophysis } \\
\text { and small } \\
\text { neurohypophysis }\end{array}$ & $\begin{array}{l}\text { hearing } \\
\text { loss (2y) }\end{array}$ & $\begin{array}{c}33 \mathrm{y} \\
\text { No ID }\end{array}$ & $\begin{array}{c}\text { Homozygous } \\
\text { c.2726C }>\mathrm{T} \\
\text { (p.Pro909Leu) }\end{array}$ & $\begin{array}{c}\text { Dysmorphic } \\
\text { features. } \\
\text { Type } 2 \\
\text { achalasia }\end{array}$ \\
\hline & 2 & $\mathrm{~F}$ & 1 & CAGSSS & Birth & $\begin{array}{l}\text { DD, distal } \\
\text { sensory } \\
\text { neuropathy } \\
(8 \mathrm{~m})\end{array}$ & $\begin{array}{l}\text { Cataract G } \\
(3 \mathrm{~m}), \\
\text { corneal } \\
\text { opacification } \\
\text { (17y) }\end{array}$ & $\begin{array}{l}\text { GH deficiency } \\
\text { (4 y) } \\
\text { Short d } \\
\text { stature }\end{array}$ & $\begin{array}{l}\text { Bilateral } \\
\text { hip } \\
\text { dislocation, } \\
\text { SEMD } \\
(18 \mathrm{~m})\end{array}$ & & $\begin{array}{l}\text { Hearing } \\
\text { loss }\end{array}$ & $\begin{array}{c}6 \mathrm{y} \\
\text { No ID }\end{array}$ & $\begin{array}{c}\text { Homozygous } \\
\text { c.2726C >T } \\
\text { (p.Pro909Leu) }\end{array}$ & $\begin{array}{c}\text { Dysmorphic } \\
\text { features }\end{array}$ \\
\hline & 3 & M & 1 & CAGSSS & Birth & $\begin{array}{c}\text { DD, } \\
\text { distal sensory } \\
\text { neuropathy }\end{array}$ & $\begin{array}{l}\text { Cataract }(5 \mathrm{~m}), \mathrm{G} \\
\text { corneal } \\
\text { opacifications } \\
(5 \mathrm{y})\end{array}$ & $\begin{array}{c}\text { GH deficiency } \\
(5 \text { y) } \\
\text { Short } \\
\text { stature }\end{array}$ & $\begin{array}{c}\text { cyBilateral hi } \\
\text { dislocation } \\
\text { (at birth), } \\
\text { SEMD }\end{array}$ & & $\begin{array}{c}\text { Hearing } \\
\text { loss }(21 \mathrm{~m})\end{array}$ & $\begin{array}{c}17 y \\
\text { No ID }\end{array}$ & $\begin{array}{c}\text { Homozygous } \\
\text { c.2726C >T } \\
\text { (p.Pro909Leu) }\end{array}$ & $\begin{array}{c}\text { Dysmorphic } \\
\text { features }\end{array}$ \\
\hline & 4 & M & 2 & $\begin{array}{l}\text { Leigh } \\
\text { syndrome }\end{array}$ & $1 \mathrm{~m}$ & No & No & No & No & $\begin{array}{c}\text { Diffuse } \\
\text { atrophy and } \\
\text { hyperintensities } \\
\text { on T2 in the basal } \\
\text { ganglia and thalami }\end{array}$ & No & $\begin{array}{c}18 \mathrm{~m} \\
\text { (deceased) }\end{array}$ & $\begin{array}{c}\text { Compound } \\
\text { heterozygous } \\
\text { c.1821G }>\text { A } \\
\text { (p.Trp607*) } \\
\text { and c.2122G }>\mathrm{A}\end{array}$ & $\begin{array}{l}\text { Increased } \\
\text { CSF L }\end{array}$ \\
\hline $\begin{array}{l}\text { Moosa et al., } \\
2017\end{array}$ & 1 & $\mathrm{~F}$ & 1 & CAGSSS & Birth & $\begin{array}{l}\text { Hypotonia, } \\
\text { peripheral } \\
\text { neuropathy }\end{array}$ & $\begin{array}{l}\text { Cataract } \\
(3 \mathrm{y})\end{array}$ & $\begin{array}{r}\text { Normal GH } \\
\text { Short stature } \\
(-6 \mathrm{SD}) \\
\text { SE }\end{array}$ & $\begin{array}{l}\text { Bilateral } \\
\text { e hip } \\
\text { dislocation } \\
\text { (at birth), } \\
\text { SEMD (18 m) }\end{array}$ & $\begin{array}{c}\text { Mild } \\
\text { hydrocephalus } \\
\text { and narrow } \\
\text { foramen } \\
\text { magnum }\end{array}$ & $\begin{array}{l}\text { Hearing } \\
\text { loss }(8 \mathrm{y})\end{array}$ & $\begin{array}{c}8 \mathrm{y} \\
\text { No ID }\end{array}$ & $\begin{array}{c}\text { (p.Glu708Lys) } \\
\text { Homozygous } \\
\text { c.2620G>A } \\
\text { (p.Gly874Arg) }\end{array}$ & $\begin{array}{l}\text { Dysmorphic } \\
\text { features } \\
\text { Telangiectasia }\end{array}$ \\
\hline \multirow[t]{2}{*}{$\begin{array}{l}\text { Takezawa et al., } \\
2018\end{array}$} & 1 & $\mathrm{~F}$ & 1 & $\begin{array}{l}\text { CAGSSS, } \\
\text { Leigh and West } \\
\text { syndrome }\end{array}$ & $5 \mathrm{~m}$ & $\begin{array}{c}\text { Infantile } \\
\text { spasms, DD }\end{array}$ & $\begin{array}{l}\text { Cataract } \\
(8 \mathrm{y})\end{array}$ & $\begin{array}{l}\text { Short } \\
\text { stature } \\
(-5 \mathrm{SD})\end{array}$ & $\begin{array}{l}\text { NK } \\
\text { se } \\
\text { and } \\
\mathrm{r} \\
\text { bas }\end{array}$ & $\begin{array}{l}\text { Mild cortical } \\
\text { atrophy (8 m); } \\
\text { evere cortical atrophy } \\
\text { d atrophy and bilateral } \\
\text { hyperintensity in the } \\
\text { sal ganglia on T2 (21 m) }\end{array}$ & $\begin{array}{r}\text { NK } \\
\mathrm{qu} \\
\text { and } \\
\text { and } \\
\text { (p }\end{array}$ & $\begin{array}{c}8 \mathrm{y} \\
\text { Hypotonic } \\
\text { quadriplegia } \\
\text { ad bedridden } \\
\text { dd c.2450G }>\text { A } \\
\text { (p.Arg817His) }\end{array}$ & $\begin{array}{c}\text { Compound } \\
\text { heterozygous } \\
\text { c.680T>C } \\
\text { (p.Phe227Ser) }\end{array}$ & $\begin{array}{l}\text { Increased } \\
\text { L and L/P } \\
\text { in blood } \\
\text { and CSF }\end{array}$ \\
\hline & 2 & $\mathrm{~F}$ & 1 & $\begin{array}{l}\text { CAGSSS, } \\
\text { Leigh and West } \\
\text { syndrome }\end{array}$ & $7 \mathrm{~m}$ & $\begin{array}{l}\text { Infantile } \\
\text { spasms }\end{array}$ & No & $\begin{array}{c}\text { NK } \\
\text { Short stature } \\
(-5 \text { SD })\end{array}$ & NK & $\begin{array}{l}\text { Diffuse cortical } \\
\text { atrophy }(7 \mathrm{~m}) \\
\text { bilateral } \\
\text { hyperintensity } \\
\text { in the basal ganglia } \\
\text { on T2 }(18 \mathrm{~m})\end{array}$ & $\begin{array}{r}\text { NK } \\
\text { qu } \\
\text { b }\end{array}$ & $\begin{array}{l}\quad 5 y \\
\text { Hypotonic } \\
\text { quadriplegia, } \\
\text { bedridden } \\
\text { (1 y) }\end{array}$ & $\begin{array}{c}\text { Compound } \\
\text { heterozygous } \\
\text { c.680T>C } \\
\text { (p.Phe227Ser) } \\
\text { and c.2450G >A } \\
\text { (p.Arg817His) }\end{array}$ & $\begin{array}{l}\text { Increased } \\
\mathrm{L} \text { and L/P } \\
\text { in blood } \\
\text { and CSF }\end{array}$ \\
\hline \multirow[t]{3}{*}{ Vona et al., 2018} & 1 & $\mathrm{M}$ & 1 & CAGSSS & Birth & $\begin{array}{l}\text { Spasticity } \\
\text { ca }\end{array}$ & $\begin{array}{c}\text { Congenital } \\
\text { ataract, nystagmus } \\
\text { corneal S } \\
\text { opacification }\end{array}$ & $\begin{array}{c}\text { GH } \\
\text { Is,deficiency } \\
\text { Short stature } \\
(-3.5 \mathrm{SD})\end{array}$ & $\begin{array}{l}\text { SEMD } \\
(18 \mathrm{y}) \\
\mathrm{e}^{-}\end{array}$ & Normal & $\begin{array}{c}\text { Hearing } \\
\text { loss }(13 \mathrm{y})\end{array}$ & $\begin{array}{c}20 \mathrm{y} \\
\text { No ID }\end{array}$ & $\begin{array}{c}\text { Homozygous } \\
\text { c.2725C > T } \\
\text { (p.Pro909Ser) }\end{array}$ & $\begin{array}{c}\text { Type } 2 \\
\text { achalasia, } \\
\text { dysmorphic } \\
\text { features }\end{array}$ \\
\hline & 2 & $\mathrm{~F}$ & 2 & CAGSSS & Birth & No & $\begin{array}{c}\text { Congenital } \\
\text { cataract, corneal } \\
\text { opacification }\end{array}$ & No & SEMD & NK & No & $\begin{array}{c}35 \mathrm{y} \\
\text { No ID }\end{array}$ & $\begin{array}{l}\text { Homozygous } \\
\text { c.2282A > G } \\
\text { (p.His761Arg) }\end{array}$ & $\begin{array}{c}\text { Dysmorphic } \\
\text { features }\end{array}$ \\
\hline & 3 & $\mathrm{~F}$ & 2 & CAGSSS & Birth & No & Congenital cataract & No & NK & NK & No & $\begin{array}{c}27 \mathrm{y} \\
\text { No ID }\end{array}$ & $\begin{array}{c}\text { Homozygous } \\
\text { c.2282A > G } \\
\text { (p.His761Arg) }\end{array}$ & $\begin{array}{c}\text { Dysmorphic } \\
\text { features }\end{array}$ \\
\hline \multirow[t]{2}{*}{ Li et al., 2018} & 1 & M & 1 & Cataract & $5 \mathrm{~m}$ & $\begin{array}{l}\text { No } \\
(5 \mathrm{~m})\end{array}$ & Cataract & No & No & NK & No & c.2575 & $\begin{array}{l}\text { Compound } \\
\text { heterozygous } \\
\text { c.607G }>C \\
\text { (p.Gly203Arg) and } \\
5 \mathrm{~T}>\mathrm{C} \text { (p.Phe859Leu) }\end{array}$ & \\
\hline & 2 & M & 2 & Cataract & $6 \mathrm{~m}$ & $\begin{array}{l}\text { No } \\
(6 \mathrm{~m})\end{array}$ & Cataract & No & No & NK & No & NK & $\begin{array}{c}\text { Compound } \\
\text { heterozygous } \\
\text { c. } 2446 \mathrm{C}>\mathrm{T}\end{array}$ & $\begin{array}{l}\text { Increased } \\
\text { serum L }\end{array}$ \\
\hline
\end{tabular}




\begin{tabular}{|c|c|c|c|c|c|c|c|c|c|c|c|c|c|c|}
\hline & 3 & M & 2 & Cataract & NK & No & Cataract & No & No & NK & No & a & $\begin{array}{c}\text { Compound } \\
\text { heterozygous } \\
\text { c.2446C }>\mathrm{T} \\
\text { (p.Arg816*) } \\
\text { and c.2575T >C } \\
\text { (p.Phe859Leu) }\end{array}$ & \\
\hline \multirow[t]{5}{*}{ Lee et al., 2019} & 1 & $\mathrm{~F}$ & 1 & $\begin{array}{c}\text { Leigh } \\
\text { syndrome }\end{array}$ & $18 \mathrm{~m} \mathrm{D}$ & $\begin{array}{l}\text { Development } \\
\text { regression }\end{array}$ & No & NK & $\begin{array}{r}\text { No } \\
\mathrm{s} \\
\text { in th }\end{array}$ & $\begin{array}{l}\text { T2 hyperintensity } \\
\text { involving both the } \\
\text { caudate nucleus and } \\
\text { basal ganglia with } \\
\text { swelling and a lactate peak } \\
\text { he basal ganglia lesion }(42 \mathrm{~m})\end{array}$ & № & $\begin{array}{c}13 \mathrm{y} \\
\text { Walks with } \\
\text { assistance; } \\
\text { no speech } \\
\\
\\
\text { (p. }\end{array}$ & $\begin{array}{l}\text { Compound } \\
\text { heterozygous } \\
\text { c.1195A }>\text { G } \\
\text { (p.Met399Val) } \\
\text { and c.2052delT } \\
\text {.Gln685Lysfs*15) }\end{array}$ & \\
\hline & 2 & $\mathrm{~F}$ & 2 & $\begin{array}{c}\text { Leigh } \\
\text { syndrome }\end{array}$ & $11 \mathrm{~m} \mathrm{D}$ & DD, hypotonia & No & NK & $\begin{array}{l}\text { No } \\
\text { area } \\
\text { toget }\end{array}$ & $\begin{array}{l}\text { T2 hyperintensity } \\
\text { in the periaqueductal } \\
\text { and midline of the midbrain, } \\
\text { ther with diffuse brain atrophy }\end{array}$ & NA & $\begin{array}{r}16 \mathrm{~m} \\
\text { (deceased) } \\
\text { c.550G> } \\
\text { c. } 1967 \mathrm{~T}\end{array}$ & $\begin{array}{l}\text { Compound } \\
\text { heterozygous } \\
>\text { A (p.Ala184Thr) } \\
\text { 7T>C (p.Phe656Se }\end{array}$ & $\begin{array}{l}\text { NK } \\
\text { and } \\
\text { er) }\end{array}$ \\
\hline & 3 & M & 3 & $\begin{array}{c}\text { Leigh } \\
\text { syndrome }\end{array}$ & $11 \mathrm{~m}$ & DD & $\begin{array}{c}\text { Nystagmus, } \\
\text { cataract }\end{array}$ & NK & No & $\begin{array}{l}\text { T2 hyperintensity } \\
\text { in both the basal ganglia } \\
\text { (putamen) and a lactate } \\
\text { eak in the putamen }(37 \mathrm{~m} \text { ) }\end{array}$ & No & $\begin{array}{c}\text { 8y } \\
\text { Walks alone; } \\
\text { No speech } \\
\\
\text { (p. } \\
\text { al } \\
\end{array}$ & $\begin{array}{l}\text { Compound } \\
\text { heterozygous } \\
\text { c.314_318del } \\
\text { p.Val105Aspfs*7) } \\
\text { and c.2450G>A } \\
\text { (p.Arg817His) }\end{array}$ & $\begin{array}{c}\text { Increased } \\
\text { serum L }\end{array}$ \\
\hline & 4 & $\mathrm{M}$ & 4 & $\begin{array}{c}\text { Leigh } \\
\text { syndrome }\end{array}$ & ${ }^{9 \mathrm{~m}} \mathrm{Mi}$ & $\begin{array}{l}\text { DD, } \\
\text { icrocephaly, } \\
\text { Seizures } \\
\text { (spasms) }\end{array}$ & Cataract & $\begin{array}{r}\text { Short } \\
\text { stature }\end{array}$ & № & $\begin{array}{l}\text { Bilateral } \\
\text { symmetric T1- weighted } \\
\text { low and T2- hyperintensity } \\
\text { in the putamen and } \\
\text { lelayed myelination }(22 \mathrm{~m})\end{array}$ & № & $\begin{array}{l}\text { 3y } \\
\text { Sit up and stand h } \\
\text { with assistance, } \\
\text { no speech } \\
\text { an }\end{array}$ & $\begin{array}{c}\text { Compound } \\
\text { heterozygous } \\
\text { c.971_972del } \\
\text { (p.Ser324*) } \\
\text { and c.2450G>A } \\
\text { (p.Arg817His) }\end{array}$ & $\begin{array}{c}\text { Increased } \\
\text { serum L }\end{array}$ \\
\hline & 5 & M & 5 & $\begin{array}{c}\text { Leigh } \\
\text { syndrome }\end{array}$ & Mi & $\begin{array}{l}\text { DD, } \\
\text { icrocephaly, } \\
\text { seizures }\end{array}$ & $\begin{array}{r}\text { Cataract } \mathrm{I} \\
\mathrm{t} \\
\mathrm{Sh}\end{array}$ & $\begin{array}{l}\text { Hypopar } \\
\text { thyroidis } \\
\text { hort stat }\end{array}$ & $\begin{array}{l}\text { - No } \\
\text { Ire } \\
\text { the } p \\
\text { diffu }\end{array}$ & $\begin{array}{c}\text { Bilateral } \\
\text { symmetric T2 } \\
\text { hyperintensity and } \\
\text { atrophic changes in both } \\
\text { putamen and caudate nucleus } \\
\text { use cerebral atrophy, and loss } \\
\text { of WM volume }\end{array}$ & NK & 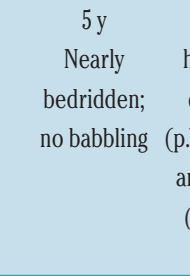 & $\begin{array}{l}\text { Compound } \\
\text { heterozygous } \\
\text { c.314_318del } \\
\text { p.Val105Aspfs*7) } \\
\text { and c.2450G }>\text { A } \\
\text { (p.Arg817His) }\end{array}$ & $\begin{array}{c}\text { Increased } \\
\text { serum L }\end{array}$ \\
\hline \multirow[t]{4}{*}{ This article } & 1 & $\mathrm{M}$ & 1 & $\begin{array}{l}\text { Leigh } \\
\text { syndrome, } \\
\text { anemia }\end{array}$ & Birth & $\begin{array}{c}\text { DD, } \\
\text { seizures } \\
\text { (spasms) }\end{array}$ & Cataract $\frac{1}{\mathrm{t}}$ & $\begin{array}{l}\text { Hypopar } \\
\text { thyroidis }\end{array}$ & & $\begin{array}{c}\text { Bilateral basal } \\
\text { ganglia hyperintensity } \\
\text { on T2-weighted } \\
\text { images }\end{array}$ & № & $\begin{array}{r}16 \mathrm{~m} \\
\text { (deceased) } \\
\text { c. } 891 \\
\text { c. } 24500\end{array}$ & $\begin{array}{c}\text { Compound } \\
\text { heterozygous } \\
\text { 91G }>\text { A (p.Trp297* } \\
0 \mathrm{G}>\mathrm{A} \text { (p.Arg817Hi }\end{array}$ & $\begin{array}{l}\text { Increased } \\
\text { serum and } \\
\text { CSF L } \\
\text { lis) }\end{array}$ \\
\hline & 2 & $\mathrm{M}$ & $\begin{array}{l}2 \\
c\end{array}$ & $\begin{array}{l}\text { Anemia, } \\
\text { cardiomyopathy }\end{array}$ & $\begin{array}{l}\text { Birth } \\
y\end{array}$ & DD & No & No & № & $\begin{array}{c}\text { Bilateral } \\
\text { basal ganglia } \\
\text { hyperintensity on } \\
\text { T2-weighted images }(1 \mathrm{~m})\end{array}$ & № & $\begin{array}{c}2 \mathrm{~m} \\
\text { (deceased) }\end{array}$ & $\begin{array}{c}\text { Homozygous } \\
\text { c.199C }>\mathrm{T} \\
\text { (p.Pro67Ser) }\end{array}$ & $\begin{array}{c}\text { Increased } \\
\text { serum L }\end{array}$ \\
\hline & 3 & $\mathrm{M}$ & $\begin{array}{l}3 \\
c\end{array}$ & $\begin{array}{l}\text { Anemia, } \\
\text { cardiomyopathy }\end{array}$ & $\begin{array}{l}\text { Birth } \\
\text { y } \\
\text { carc }\end{array}$ & $\begin{array}{l}\text { DD, } \\
\text { Anemia, } \\
\text { diomyopathy }\end{array}$ & $\begin{array}{c}\text { Cataract } \\
(4,5 \mathrm{~m}) \\
\text { Glaucoma } \\
4 \mathrm{y}\end{array}$ & No & $\begin{array}{r}\text { coliosis } \\
\qquad \mathrm{St} \\
\text { an } \\
\text { with } \\
\text { spec }\end{array}$ & $\begin{array}{l}\text { bilateral caudate } \\
\text { nuclei and putamen } \\
\text { hyperintensities }(16 \mathrm{~m}) \text {. } \\
\text { ubsequent global cerebral } \\
\text { ad basal ganglia volume loss } \\
\text { h a small lactate peak on MR } \\
\text { ctroscopy in the basal ganglia. }\end{array}$ & № & $\begin{array}{c}14 \mathrm{y} \\
\text { (deceased), } \\
\text { profound IDc.20 } \\
\text { (no walking, } \\
\text { no language), } \\
\text { pharmaco-resistant } \\
\text { epilepsy, NGF }\end{array}$ & $\begin{array}{l}\text { Compound } \\
\text { heterozygous } \\
\text { 2025dup; p.Asp } 676^{*} \\
\text { c.986T>C; } \\
\text { p.Leu329 Pro } \\
\text { t }\end{array}$ & $\begin{array}{l}\text { Intermittently } \\
\text { increased } \\
* \quad \text { serum L }\end{array}$ \\
\hline & 4 & $\mathrm{~F}$ & 3 & $\begin{array}{l}\text { DD, right } \\
\text { mild } \\
\text { hemiplegia }\end{array}$ & Birth & $\begin{array}{c}\text { DD, } \\
\text { Seizures } \\
\text { (spasms), }\end{array}$ & $\begin{array}{l}\text { Cataract } \\
(6 \mathrm{~m}) \\
\text { Glaucoma } \\
26 \mathrm{y}\end{array}$ & No & coliosis & $\begin{array}{c}\text { Volume loss } \\
\text { involving the caudate } \\
\text { nuclei, globus pallidi } \\
\text { and putamina. T2 flair } \\
\text { hyperintensities involving } \\
\text { the basal ganglia }\end{array}$ & No & $\begin{array}{c}26 \text { y, profound } \\
\text { ID (no walking, } \\
\text { no language), } \\
\text { pharmaco-resistant } \\
\text { seizures. }\end{array}$ & $\begin{array}{c}\text { Compound } \\
\text { heterozygous } \\
\text { c.2025dup; } \\
\text { p.Asp676* } \\
\text { c.986T>C; } \\
\text { p.Leu329 Pro }\end{array}$ & $\begin{array}{l}\text { Increased } \\
\text { serum } \\
\text { and CSF L }\end{array}$ \\
\hline
\end{tabular}


A
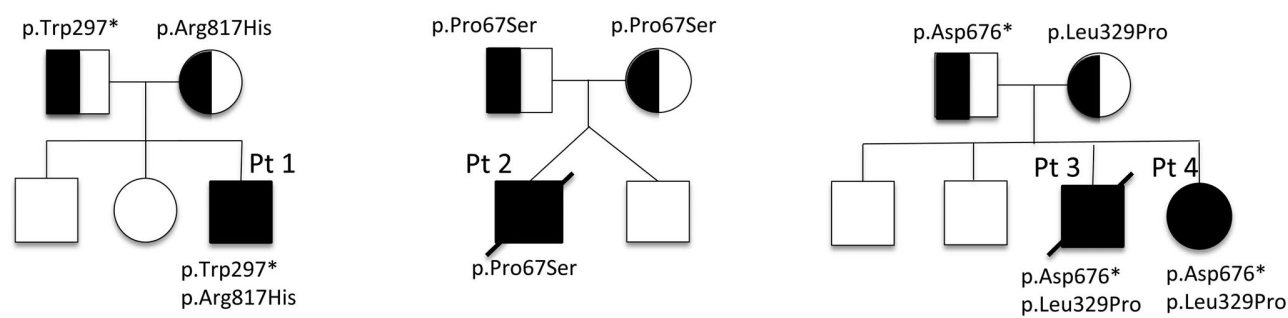

B

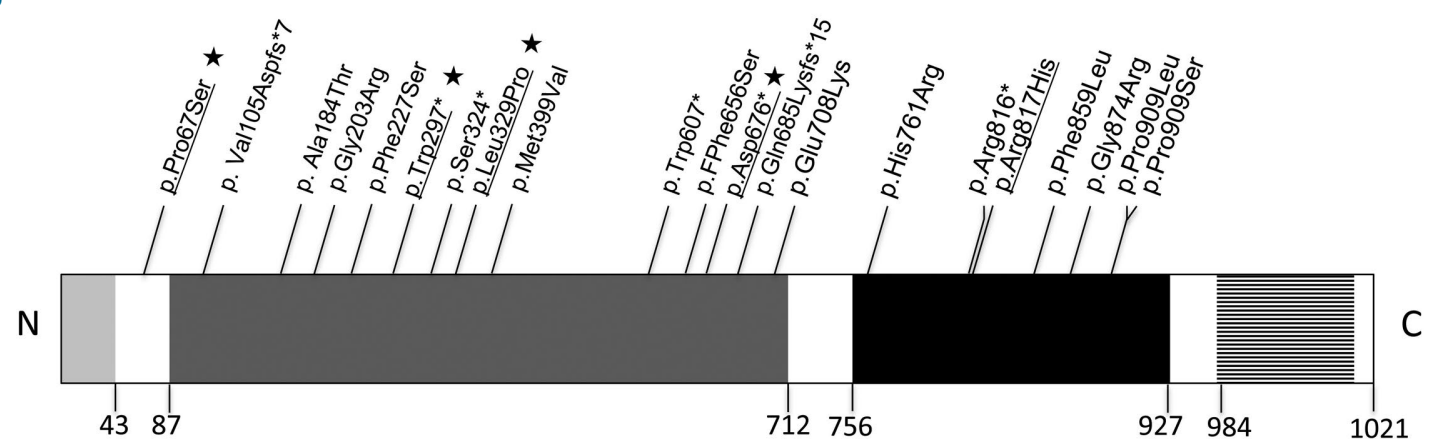

Mitochondrial leader peptide

Anticodon binding domain

tRNA synthetase domain

FPG lleRS Zinc finger domain

Figure 2. Pedigrees of the reported families and pathogenic variants described in the IARS2 gene. (A) Pedigrees, segregation and localization of the pathogenic IARS2 variants. (B) Linear map of the reported pathogenic IARS2 variants. Important structural domains and their locations on the protein map are shown. Amino acid location of each reported pathogenic variant is indicated (NM_018060.3). The likely pathogenic variants reported in this article are underlined and, if novel, indicated by a star.

the anticodon binding domain, and a missense variant inherited from his mother (c.2450G>A, p. Arg817His). The p. Arg817His variant has been previously reported in four patients with Leigh syndrome. ${ }^{5,8}$

Patient 2 was born from non-consanguineous healthy parents from Sri Lanka, at 31 weeks of gestation by cesarean section for cardiac rhythm anomalies after a dichorionic twin pregnancy (Apgar score 3, 6 and 7). His brother had no fetal distress and was healthy. He presented with respiratory distress and anemia at birth (hemoglobin 3.9 g/dL, normal 9-14 g/dL; MCV 84 fl). He needed transfusions at days 54, 69, 76 and 79 . Thrombocytopenia was also present at birth (115 platelets $/ \mathrm{mm}^{3}$ with a nadir at 50 , normal $\left.>150.000\right)$ and myelogram showed $3.7 \%$ of ringed sideroblasts (Figure $1 \mathrm{E})$. His plasma lactate was elevated (3.6-9.7 mmol/L, normal $<2 \mathrm{mmol} / \mathrm{L})$. A complete metabolic work-up, including plasma amino acids and vitamin B12 and urinary organic acids was normal. Brain MRI showed mild, diffuse white matter hyperintensities (Figure 1B). He was referred to the intensive care unit for mechanical ventilation. At day 54, he presented with pericardial effusion in the context of severe biventricular hypertrophic cardiomyopathy. He died at 2,5 months due to bradycardia.

Whole exome sequencing identified a homozygous variant in the IARS2 gene (c.199C>T; p. Pro67Ser). This variant, absent in publicly available databases, is highly conserved through evolution and predicted to be damaging by in silico softwares (Figure 1B).

Patient 3, the last of four siblings, was born at 33 weeks gestation by emergency caesarean section due to reduced fetal movements (Apgar score 1, 5 and 6). His elder sister (patient 4, see pedigree in Figure 2) had a mild left intra-ventricular bleed on an early cranial ultrasound and was diagnosed with bilateral cataracts at 6 months. She was found to have global developmental delay and infantile spasms at 32 months of age with hypsarrhythmia on EEG. Her MRI neuroimaging showed volume loss involving caudate nuclei, globi pallidi and putamina, T2 flair hyperintensities of basal ganglia with no evidence of lactate doublet on nuclear magnetic resonance (NMR) spectroscopy. No cardiac or hematological involvement were noted and her bone marrow biopsy was unremarkable.

Patient 3 required ventilation for 2 weeks and developed profound anemia (hemoglobin nadir $3.1 \mathrm{~g} / \mathrm{dL}$ ) and severe cardiomegaly with poor cardiac function. At 4.5 months (11 weeks corrected), he was diagnosed with bilateral cataracts. At 18 months of age, he presented with a first metabolic crisis in the context of pneumonia associated with profound hypotonia, severe anemia and elevated lactate. He developed persistent oropharyngeal incoordination that necessitated gastrostomy. At 2.5 years, he developed refractory tonic seizures and an EEG demonstrated frequent bilateral spike-wave discharges. He was able to smile and acquired head control but was unable to sit unsupported.

At 4 years of age, the transfusion-dependent anaemia relapsed (hemoglobin nadir $3.5 \mathrm{~g} / \mathrm{dL}$ ). Bone marrow aspiration showed subnormal myelopoiesis and megakaryopoiesis but marked erythroid hypoplasia and dysplasia with an increased number of progenitors, vacuolation 
and basophilic stippling. Iron staining showed ring sideroblasts in $10 \%$ of erythroid cells. He went on to develop mild concentric left ventricular hypertrophy, glaucoma, constipation, scoliosis and mixed central/obstructive sleep apnoea requiring non-invasive ventilation therapy. Brain MRI at 16 months of age demonstrated bilateral hyperintensities of caudate nuclei and putamen. Subsequent neuroimaging showed global cerebral and basal ganglia volume loss with bilateral putamina cavitation, characteristic of Leigh disease and a small lactate peak on NMR spectroscopy in basal ganglia (Figure 1C). Plasma lactate was intermittently elevated (up to 3.3 $\mathrm{mmol} / \mathrm{L}$, normal 0.6-2.4 mmol/L) but biochemical workup was otherwise normal. He died from central respiratory failure at the age of 14 years. In patient 3 and 4 , whole exome sequencing identified two compound heterozygous variants in IARS2; a paternally inherited nonsense variant (c.2025dup; p. Asp676*) predicted to result in either NMD or loss of the terminal two thirds of the protein including anticodon binding domain; and a maternally inherited missense variant (c.986T >C; p. Leu329Pro) modifying a mildly conserved residue. Neither of these variants have been reported to date.

Respiratory chain enzyme activities were normal in the liver, skeletal muscle or lymphocytes of patients 1 and 3 (Table). Blue native polyacrylamide gel electrophoresis of respiratory enzyme chain complexes was normal in cultured skin fibroblasts of patient 1 (not shown). No mitochondrial DNA (mtDNA) deletions or rearrangements were found in circulating leukocytes and bone marrow of patients 1 to 3 (Table).

Here, we report on three unrelated patients presenting with sideroblastic anemia, initially suggestive of Pearson Marrow-Pancreas syndrome. The absence of mtDNA deletion or complex rearrangements prompted to reconsider this diagnosis and to eventually identify biallelic pathogenic IARS2 variants in the three patients. Children with Pearson syndrome usually present with bone marrow failure and exocrine pancreatic dysfunction in the first year of life. They have macrocytic sideroblastic anemia with ringed sideroblasts detected by iron staining of the bone marrow. This transfusion-dependent condition is accompanied by thrombocytopenia and neutropenia. The disease gradually worsens and multisystem involvement occurs, including failure to thrive, liver failure, hypotonia and lactic acidosis. Survival and spontaneous recovery from bone marrow dysfunction after several years is possible, with a transition to clinical manifestations of Kearns-Sayre syndrome. ${ }^{9}$ Pearson syndrome is caused by a single large-scale mitochondrial DNA deletion. ${ }^{10}$

At variance with Pearson syndrome, our patients had a low level of ring sideroblasts in blood marrow aspiration, and presented with an early extra-hematological involvement (cardiomyopathy, cataract, and neurological involvement).

Pearson syndrome is not the unique cause of sideroblastic anemia in respiratory chain deficiency. In fact, sideroblastic anemia has been associated with pathogenic variants in other mitochondrial proteins, namely SLC25A38, PUS1, ABCB7, GLRX5, NDUFB11, COX10, HSPA9, TRNT1 and ATP6. ${ }^{11}$ Moreover, congenital sideroblastic anemia has been associated with mutations in two other mitochondrial ARS2 genes, namely YARS2 and LARS2. ${ }^{12-14}$

IARS2 mutations were first identified in patients with CAGSSS, then in patients with Leigh syndrome, and more recently in patients with cataract. To date, 18 patients have been reported with a broad range of partial- ly overlapping symptoms (Table 1$) \cdot^{3-8}$

Our report expands the clinical spectrum of IARS2related disorders to early-onset sideroblastic anemia mimicking Pearson syndrome. It adds IARS2 to the list of mitochondrial disease genes underlying sideroblastic anemia in early childhood. Future studies will hopefully help in identifying the actual impact of respiratory chain deficiency on human erythropoiesis and explaining why sideroblastic anemia is a frequent, yet inconstant feature in mitochondrial disorders.

Giulia Barcia, ${ }^{1}$ Dinusha Pandithan, ${ }^{2}$ Benedetta Ruzzenente, ${ }^{3}$ Zahra Assouline, ${ }^{1}$ Alessandra Pennisi, ${ }^{1}$ Clothilde Ormieres, ${ }^{1}$ Claude Besmond, ${ }^{4}$ Charles-Joris Roux, ${ }^{5}$ Nathalie Boddaert, Isabelle Desguerre, ${ }^{6}$ David R. Thorburn, 7,8 Drago Bratkovic, Arnold Munnich, ${ }^{1-3}$ Jean-Paul Bonnefont, ${ }^{1-3}$ Agnès Rötig ${ }^{3}$ and Julie Steffann $n^{1-3}$

"Federation of Medical Genetics and Reference Center for Mitochondrial Diseases (CARAMMEL), Hospital Necker - Enfants Malades, Paris, France; 'Metabolic Clinic, Women's and Children's Hospital, North Adelaide, South Australia, Australia; ${ }^{3}$ Laboratory for Genetics of Mitochondrial Disorders, UMR 1163, Université de Paris, Institut Imagine, Paris, France; ${ }^{4}$ Translational Genetics Laboratory, UMR U1163, Institut Imagine, Université Paris Descartes-Sorbonne Paris Cité, Paris, France; 'Department of Pediatric Radiology, Hospital Necker Enfants Malades, Paris, France; ${ }^{6}$ Department of Pediatric Neurology, Hospital Necker-Enfants Malades, Paris, France; ${ }^{7}$ Murdoch Children's Research Institute and Victorian Clinical Genetics Services, Royal Children's Hospital, Melbourne, Victoria, Australia and ${ }^{8}$ Department of Pediatrics, University of Melbourne, Melbourne, Victoria, Australia

\section{Correspondence: GIULIA BARCIA - giulia.barcia@aphp.fr}

\section{doi:10.3324/haematol.2020.270710}

\section{Disclosures: no conflicts of interest to disclose.}

Contributions: GB performed molecular researchs, data analysis and wrote the manuscript; $A M$ supervised this study, performed clinical evaluation, and wrote the manuscript. BR performed data analysis; $Z A$ and $C B$ performed the molecular study; DP, AP, CO, ID performed clinical follow-up; CJR and NB performed neuro-imaging analysis; $D R T, D B, J P B, A R$, JS performed data analysis and supervised the study.

\section{References}

1. Meyer-Schuman R, Antonellis A. Emerging mechanisms of aminoacyl-tRNA synthetase mutations in recessive and dominant human disease. Hum Mol Genet. 2017;26(R2):R114-R127.

2. Konovalova S, Tyynismaa H. 2013. Mitochondrial aminoacyl-tRNA synthetases in human disease. Mol Genet Metab. 2013;108(4):206211.

3. Schwartzentruber J, Buhas D, Majewski J, et al. Mutation in the nuclear-encoded mitochondrial isoleucyl-tRNA synthetase IARS2 in patients with cataracts, growth hormone deficiency with short stature, partial sensorineural deafness, and peripheral neuropathy or with Leigh syndrome. Hum Mutat. 2014;35(11):1285-1289.

4. Moosa S, Haagerup A, Gregersen PA, et al. Confirmation of CAGSSS syndrome as a distinct entity in a Danish patient with a novel homozygous mutation in IARS2. Am J Med Genet A. 2017; 173(4):1102-1108

5. Takezawa Y, Fujie H, Kikuchi A, et al. Novel IARS2 mutations in Japanese siblings with CAGSSS, Leigh, and West syndrome. Brain Dev. 2018;40(10):934-938

6. Vona B, Maroofian R, Bellacchio E, et al. Expanding the clinical phenotype of IARS2-related mitochondrial disease. BMC Med Genet. 2018;19(1):196

7. Li J, Leng Y, Han S, et al. Clinical and genetic characteristics of Chinese patients with familial or sporadic pediatric cataract. Orphanet J Rare Dis. 2018;13(1):94.

8. Lee JS, Kim MJ, Kim SY, et al. Clinical and genetic characteristics of Korean patients with IARS2 related disorders. J Genet Med. 2019;16(2):55-61

9. Pearson HA, Lobel JS, Kocoshis SA, et al. A new syndrome of refrac- 
tory sideroblastic anemia with vacuolization of marrow precursors and exocrine pancreatic dysfunction. J Pediatr. 1979;95(6):976-984.

10. Rotig A, Colonna M, Bonnefont P, et al. Mitochondrial DNA deletion in Pearson's marrow/pancreas syndrome. Lancet. 1989; 333 (8643):902-903.

11. Tesarova M, Vondrackova A, Stufkova H, et al. Sideroblastic anemia associated with multisystem mitochondrial disorders. Pediatr Blood Cancer. 2019;66(4):e27591

12. Riley LG, Cooper S, Hickey P, et al. Mutation of the mitochondrial
tyrosyl-tRNA synthetase gene, YARS2, causes myopathy, lactic acidosis, and sideroblastic anemia-MLASA syndrome. Am J Hum Genet. 2010;87(1):52-59.

13. Riley LG, Rudinger-Thirion J, Schmitz-Abe K, et al. LARS2 variants associated with hydrops, lactic acidosis, sideroblastic anemia, and multisystem failure. JIMD Rep. 2016;28:49-57.

14. Long Z, Li H, Du Y, Han B. Congenital sideroblastic anemia: advances in gene mutations and pathophysiology. Gene. 2018; 668:182-189 